\title{
Collisionality dependence of edge rotation and in-out impurity asymmetries in ASDEX Upgrade H-mode plasmas
}

\author{
E. Viezzer ${ }^{1}$, E. Fable ${ }^{1}$, T. Pütterich ${ }^{1}$, A. Bergmann ${ }^{1}$, M.

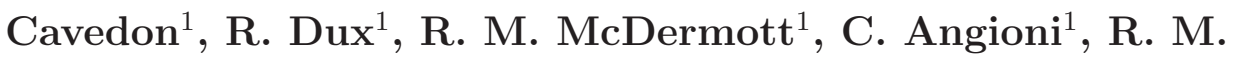 \\ Churchill $^{2}$, M. G. Dunne ${ }^{1}$, F. M. Laggner ${ }^{3}$, B. Lipschultz ${ }^{4}$, U. \\ Stroth $^{1}$, E. Wolfrum ${ }^{1}$ and the ASDEX Upgrade Team \\ 1 Max Planck Institute for Plasma Physics, Boltzmannstr. 2, 85748 Garching, \\ Germany \\ 2 MIT Plasma Science and Fusion Center, Cambridge, MA, USA \\ 3 Institute of Applied Physics, TU Wien, Fusion@ÖAW, Wiedner Hauptstr. 8-10, \\ 1040 Vienna, Austria \\ 4 University of York, Heslington, York YO10 5DD, UK \\ E-mail: Eleonora.Viezzer@ipp.mpg.de
}

\begin{abstract}
The poloidal and toroidal impurity flows in the edge transport barrier of $\mathrm{H}$-mode plasmas have been studied over a wide range of pedestal top ion collisionalities. A comparison of the edge poloidal rotation measurements to neoclassical predictions shows good agreement in all cases. The measured edge impurity toroidal rotation is observed to change sign from co-current to counter-current with decreasing collisionality. The switch occurs at the same collisionality at which neoclassical theory predicts the main ion poloidal rotation to change from the electron to the ion diamagnetic direction. The behaviour of these two species, when used to calculate the main ion toroidal rotation via the radial force balance equation, leads to fairly constant co-current main ion toroidal rotation. Hence, at low collisionality, due to a reduced frictional coupling, the main ion-impurity differential rotation can be quite large. The behaviour of impurity ion flows on a flux surface has also been investigated in detail and it was found that the measurements are consistent with the continuity equation only if the poloidally asymmetric impurity density distribution is taken into account. The asymmetry is found to be the result of the interplay of all forces in the parallel momentum balance, with the friction force providing the dominant drive. Close to the separatrix the poloidal centrifugal force, which is usually neglected, also gives an additional contribution to the impurity density asymmetry. Within the experimental uncertainties the ion temperature and the electrostatic potential are simultaneous flux functions, despite the presence of a poloidally asymmetric impurity density profile.
\end{abstract}

PACS numbers: 52.55.Fa, 52.25.Vy, 52.30.-q, 52.70.-m 


\section{Introduction}

Understanding the behaviour of impurities in the pedestal of tokamak H-mode plasmas is crucial for predicting impurity transport and the penetration into the plasma core. Turbulence often dominates the particle transport in the core of the plasma, however, there is growing evidence that in the edge pedestal, where turbulent fluctuations are strongly reduced due to $\mathbf{E} \times \mathbf{B}$ shearing [1], the impurity particle transport [2] and the ion heat transport [3] are at neoclassical levels. The electron heat transport is also reduced, but still larger than the neoclassical prediction [3].

Neoclassical theory predicts the flux-surface averaged transport fluxes and the poloidal flows of main ions and impurities as well as their relationships to the toroidal flows and the particle densities $[4,5,6]$. In tokamaks, a strong damping of the poloidal rotation, $v_{\theta}$, is present since poloidally passing ions collide with trapped ions. Since this viscous damping for a species depends on the species poloidal rotation, the poloidal flow of main ions and impurities, $v_{\theta, i}$ and $v_{\theta, z}$, can be decoupled [6]. The poloidal flow is determined by friction and the flux-surface averaged parallel stress tensor $\left\langle\mathbf{B} \cdot \nabla \cdot \boldsymbol{\Pi}_{z}\right\rangle$ if no poloidal momentum sources are present [6]. Poloidal particle density asymmetries $[7,8,9,10]$, poloidally asymmetric momentum and particle sources [11] as well as Stringer instabilities $[12,13]$ can cause a poloidal spin-up of the plasma.

Early experiments at DIII-D [14] showed that at the plasma edge $v_{\theta, i}$ and $v_{\theta, z}$ are fundamentally different, both in magnitude and in sign, with $v_{\theta, i}$ being in the ion diamagnetic drift direction while the impurities rotate in the electron diamagnetic direction. Comparison to neoclassical predictions showed that the magnitude of the simulated $v_{\theta, i}$ did not agree with the measured profile. The main ion poloidal flow was measured using charge exchange recombination spectroscopy (CXRS) on $\mathrm{He}^{2+}$ in helium plasmas and yielded velocities of up to $+40 \mathrm{~km} / \mathrm{s}$ at the plasma edge, while the neoclassical prediction was between $0-2 \mathrm{~km} / \mathrm{s}$. However, comparison of the measured DIII-D data with neoclassical predictions including the orbit-squeezing effect [15], i.e. the ion banana widths are reduced due to the gradient in the radial electric field [16], showed a fair agreement at the very far edge of the plasma. However, large discrepancies were still observed $\sim 1-3 \mathrm{~cm}$ inside the plasma [15].

Poloidal rotation measurements have been compared to neoclassical predictions on many tokamaks, however, they have yielded very different results. A mismatch with neoclassical theory was observed in internal transport barriers formed in enhanced reversed shear discharges at TFTR [17], in the core of H-mode and quiescent H-mode discharges at DIII-D [18] as well as in internal transport barriers at JET [19]. Recent observations at DIII-D based on CXRS measurements of the impurity and main ion toroidal rotation, $v_{\phi, z}$ and $v_{\phi, i},[20,21]$ revealed that in plasmas with low collisionality the core deuterium poloidal flow disagrees with neoclassical theory, being more in the ion diamagnetic drift direction in the experiment. In other cases the measurements showed good agreement with neoclassical theory within the experimental uncertainties $[22,23,7,24,25,26]$ including direct measurements of the main ions in the highly 
collisional edge pedestal [27].

To shed more light on the physics determining the poloidal flow in a tokamak plasma, an edge rotation database was created at ASDEX Upgrade (AUG) including measurements at both low and high collisionality. The edge rotation database is introduced in section 2 and example profiles are shown. The poloidal rotation measurements are compared to neoclassical predictions using the NEOART code [28] (see section 3). In section 4 the measured poloidal impurity asymmetries are compared to neoclassical predictions using the fluid model, that has been developed to quantify the in-out impurity asymmetries, and the kinetic code HAGIS $[29,30]$. Details are given to quantify the dominant driving terms responsible for the poloidally asymmetric impurity distributions and to study their impact on the edge transport barrier. A summary and the main conclusions of the paper are given in section 5 .

\section{Extension of measurements to the low collisionality regime}

An edge rotation database was compiled at AUG to test whether neoclassical theory can predict the measured $v_{\theta}$ in the edge of $\mathrm{H}$-mode plasmas over a wide range of collisionality. Previous work at AUG showed that in the highly collisional edge pedestal of H-mode plasmas, with the main ion collisionality $\nu_{*, i}$ in the pedestal being in the plateau or the Pfirsch-Schlüter regime, the edge poloidal flow is consistent with neoclassical predictions [27]. The parameter range of the discharges included in the database are listed in table 1. The main ion collisionality used here is defined as the effective collision frequency normalized to the trapped particle bounce frequency, $\nu_{*, i}=\nu_{\text {eff }} / \omega_{\mathrm{b}}=\nu q R /\left(\epsilon^{3 / 2} v_{t h}\right)$. Note that here collisions between all species are taken into account. The collision frequency for momentum transfer from species $j$ to $k$ is $\nu_{j k}=\frac{4 \sqrt{2 \pi}}{3\left(4 \pi \epsilon_{0}\right)^{2}} \frac{\sqrt{m_{j k}}}{m_{j}} \frac{q_{j}^{2} q_{k}^{2} \ln \Lambda_{j k}}{\left(k_{B} T\right)^{3 / 2}} n_{k}$, where $\ln \left(\Lambda_{j k}\right)$ is the Coulomb logarithm and $m_{j k}$ is the reduced mass of species $j$ and $k$. Using this definition, the banana regime is defined for $\nu_{*, i} \ll 1$, the Pfirsch-Schlüter regime for $\nu_{*, i} \gg \epsilon^{-3 / 2}$ and the intermediate plateau regime for $1 \ll \nu_{*, i} \ll \epsilon^{-3 / 2}$. The edge collisionality at $\rho_{\text {pol }}=0.97$ in the experiment covered the range between 0.18 to 11.5. In the following, the experimental collisionality values that are below the banana

\begin{tabular}{|c|c|c|c|c|c|c|}
\hline Gas & $I_{p}[\mathrm{MA}]$ & $B_{\phi}[\mathrm{T}]$ & $q_{95}$ & $\bar{n}_{e}\left[10^{19} \mathrm{~m}^{-2}\right]$ & $\nu_{*, i}^{\text {ped,top }}$ & $\beta_{N}$ \\
\hline $\mathrm{H}$ & $0.8-1.0$ & $2.5-2.6$ & $3.8-5.2$ & $6.1-6.7$ & $0.43-2.2$ & $1.0-1.3$ \\
\hline $\mathrm{D}$ & $0.8-1.0$ & $1.8-2.5$ & $3.7-5.2$ & $4.3-8.1$ & $0.18-2.5$ & $1.1-2.9$ \\
\hline $\mathrm{He}$ & 1.0 & 2.5 & 4.4 & $9.0-10.7$ & $11.2-11.5$ & $1.3-1.4$ \\
\hline
\end{tabular}

Table 1: Parameter range for the discharges analyzed in the presented database: plasma current $I_{p}$, toroidal magnetic field on-axis $B_{\phi}$, edge safety factor $q_{95}$, core line-integrated density $\bar{n}_{e}$, main ion collisionality at the pedestal top $\nu_{*, i}^{\text {ped,top }}\left(\rho_{\text {pol }}=0.97\right)$ and normalized beta $\beta_{N}$, defined as $\beta_{N}=\beta \cdot \frac{a B_{t}}{I_{p}}$, where $\beta$ is the ratio between the plasma pressure and the magnetic pressure and $a$ the plasma minor radius. 

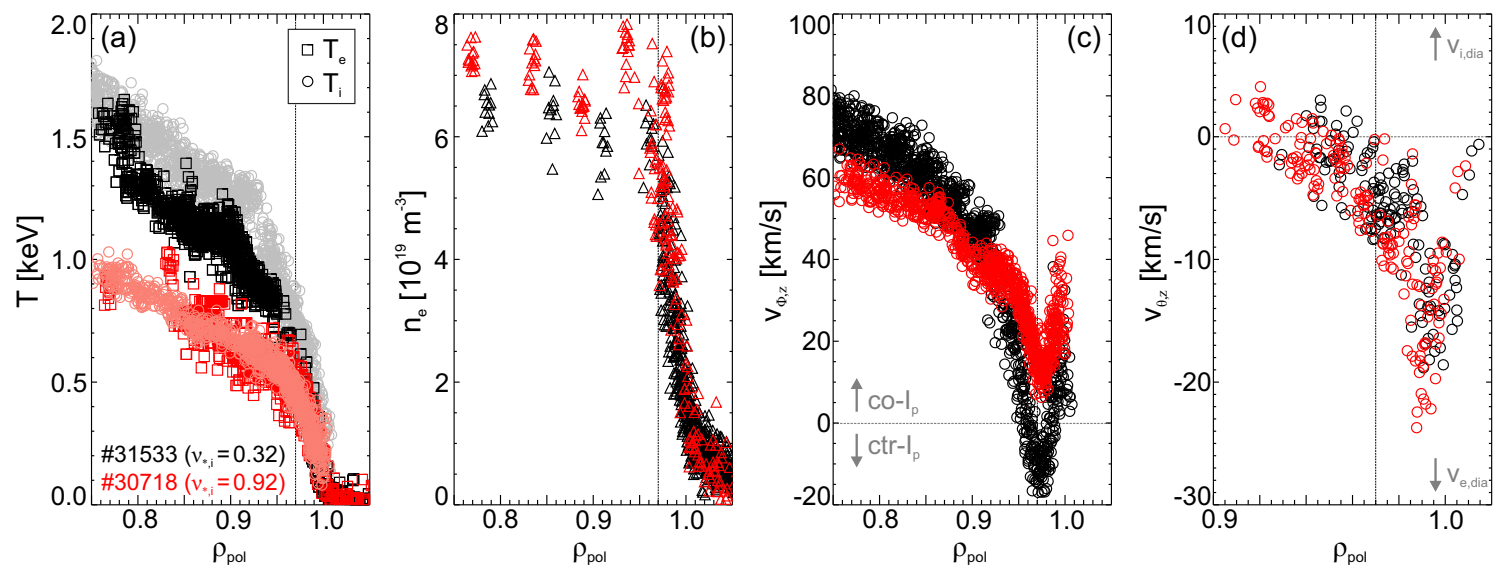

Figure 1: (a) Electron and ion temperature profiles, (b) electron density and impurity (c) toroidal rotation and (d) poloidal rotation measured in H-mode D plasmas with low $\left(\nu_{*, i}=0.32\right.$, below the banana limit $)$ and medium collisionality $\left(\nu_{*, i}=0.92\right.$, plateau regime). The values for $\nu_{*, i}$ are taken at $\rho_{p o l}=0.97$.

limit are tagged as being in the banana regime, while values above the Pfirsch-Schlüter limit are defined as being in the Pfirsch-Schlüter regime. In medium to high collisionality plasmas the coupling between electrons and ions is sufficiently high such that $T_{e} \approx T_{i}$ [31], while the ion and electron channels are less coupled at low collisionality. The pedestal top values taken at $\rho_{\text {pol }}=0.97$ for the ion temperature, electron temperature and density covered in the database ranged from $294.1-874.2 \mathrm{eV}, 329.6-797.6 \mathrm{eV}$ and $3.1-7.4 \cdot 10^{19} \mathrm{~m}^{-3}$, respectively.

Figure 1 shows example profiles of the ion and electron temperature, electron density, $v_{\phi, z}$ and $v_{\theta, z}$ measured at low $\left(\nu_{*, i}=0.32\right.$, below the banana limit $)$ and medium $\left(\nu_{*, i}=0.92\right.$, plateau regime) collisionality plasmas in deuterium (D) . The $T_{i}, v_{\phi, z}$ and $v_{\theta, z}$ (usually boron $\mathrm{B}^{5+}$ on $\mathrm{AUG}$ ) profiles are measured using the core and edge charge exchange systems [32] (note that for $v_{\theta, z}$ only an edge view is available). $T_{e}$ is measured with the electron cyclotron emission (ECE) [33] and the core and edge Thomson scattering (TS) diagnostics [34]. The electron density profiles are obtained via TS, impact excitation spectroscopy at a Lithium beam [35] and laser interferometry [36]. The vertical lines indicate the radial position $\rho_{\text {pol }}=0.97$, which is typically the pedestal top position in $n_{e}$. Note that $\rho_{p o l}$ is the normalized poloidal flux label, defined as $\rho_{p o l}=\sqrt{\frac{\Psi-\Psi_{a}}{\Psi_{s}-\Psi_{a}}}$, where $\Psi$ is the poloidal flux, $\Psi_{a}$ and $\Psi_{s}$ the poloidal flux at the magnetic axis and at the separatrix. To reduce uncertainties due to the magnetic equilibrium a relative profile alignment with respect to the separatrix position is applied [37], which has an accuracy of $2-3 \mathrm{~mm}$. The data are ELM synchronized, i.e. the measured data is sorted with respect to the onset of an ELM and taken during a phase that includes a radial plasma sweep to increase the line of sight coverage of the edge diagnostics in the pedestal region. In figure 1 only data where the full exposure time of the frame lies in the time window $[-4.5,-1.0] \mathrm{ms}$ relative to the ELM crash is used. While the electron density 
is similar in both discharges, the core electron and ion temperatures are roughly $50 \%$ higher at lower $\nu_{*, i}$. The poloidal rotation profile is also similar in both discharges and is pointing into the electron diamagnetic drift direction (negative $v_{\theta, z}$ ) at the plasma edge. Remarkably, the edge toroidal rotation flips sign at the plasma edge $\left(\rho_{\text {pol }}>0.95\right)$ and is in the counter-current direction at low collisionality (see figure 1(c)). The well in the edge toroidal rotation profile, characteristic of H-modes in AUG [38], is maintained though shifted to negative values. The feature also appears to be wider, consistent with a wider $T_{i}$ pedestal (see figure $1(\mathrm{a})$ ). The identification of the dominant mechanism driving the edge toroidal rotation at $\mathrm{AUG}$ is the subject of future work.

\section{Comparison between measured low-field side poloidal rotation and conventional neoclassical predictions}

The poloidal rotation measurements obtained at low collisionality are compared to neoclassical predictions to test whether neoclassical theory can reproduce the measurements in low collisionality plasmas.

Neoclassical theory predicts the poloidal flow of main ions and impurities using information on the plasma background profiles and the magnetic equilibrium $[4,5,6,39]$. The neoclassical main ion and impurity poloidal rotation velocities, $v_{\theta, i}^{\text {neo }}$ and $v_{\theta, z}^{\text {neo }}$, are derived from the parallel component of the momentum force balance [6]. For $v_{\theta, i}^{\text {neo }}$ the $T_{i}$ gradient is crucial, while for $v_{\theta, z}^{\text {neo }}$ also the pressure gradient scale length of the main ions is important [6].

In the edge pedestal of $\mathrm{H}$-mode plasmas, $v_{\theta, z}$ can reach large values due to the steep ion pressure gradient $\nabla p_{i}$. The impurities typically rotate in the electron diamagnetic drift direction. The main ion poloidal flow can be close to zero or even change its sign (pointing into the electron or into the ion diamagnetic drift direction), depending on the ion parameters at the plasma edge, mainly on $\nu_{*, i}$. In the following, the measurements of both $v_{\theta, z}$ and $v_{\theta, i}$ are compared to the neoclassical prediction evaluated with the NEOART code [28] for a variety of H-mode plasmas with different pedestal top collisionalities. NEOART is based on the calculation of collisional transport coefficients for a given number of impurities and includes collisions between all species. The transport coefficients represent the sum of a classical, a Pfirsch-Schlüter and a banana plateau term $[28,40]$. A set of linear coupled equations for the parallel velocities in an arbitrary toroidally symmetric geometry is solved and neoclassical transport parameters for all collisionality regimes are calculated $[28,40]$.

Figure 2(a) shows the value at the minimum in the edge poloidal flow profile of both impurities and main ions plotted as a function of $\nu_{*, i}$ at the minimum $v_{\theta}$. Note that negative values in $v_{\theta}$ are in the electron diamagnetic drift direction. Data obtained in deuterium (D) plasmas is shown in black, while hydrogen (H) and helium (He) plasmas are highlighted in red and blue, respectively. The data point marked in light purple corresponds to discharge \#28093 which is discussed in detail in section 4. Note that in He plasmas the measured $v_{\theta}$ corresponds to the main ion species, obtained via CXRS on 
$\mathrm{He}^{2+}$, while in $\mathrm{D}$ and $\mathrm{H}$ plasmas the measurements are obtained on impurity ions (boron, carbon, nitrogen or neon). The choice of the species depends on the intrinsic impurity level and if impurity seeding is applied in the experiment (such as $\mathrm{N}_{2}$, Ne or $\mathrm{CD}_{4}$ ). No direct correlation between the well in the edge poloidal impurity flow and the main ion collisionality at the same flux surface is observed; the same magnitude of poloidal rotation can be reached at different collisionality values. Figure 2(b) shows a comparison of the measured (stars) and neoclassical poloidal flow of both impurities (squares) and main ions (circles) at $\rho_{\text {pol }}=0.97$ for the pedestal top collisionality range covered in the experiment (0.18-11.5). It should be noted that in the edge transport barrier, poloidal impurity density asymmetries (see section 4) are present and therefore standard neoclassical codes, which assume that the species density is a flux function, cannot be used and modelling with 2D codes is required. Therefore, we compare the measured and neoclassical poloidal rotation at $\rho_{\text {pol }}=0.97$. There is sufficient experimental uncertainty that we cannot determine whether the in-out $n_{z}$ asymmetry at $\rho_{\text {pol }}=0.97$ is small or significant. We apply the NEOART code nonetheless, noting that it is being applied outside its range of validity if the asymmetry is indeed significant. For all cases, the experimental points are well matched by the neoclassical prediction. This shows that in the established H-mode, viscous damping is the driving mechanism for $v_{\theta, i}$ at the plasma edge, given by the $T_{i}$ gradient $\left(\nabla T_{i}\right)$. These results are also in keeping with the radial electric field, $E_{r}$, being sustained by the gradients of the main ions in the edge
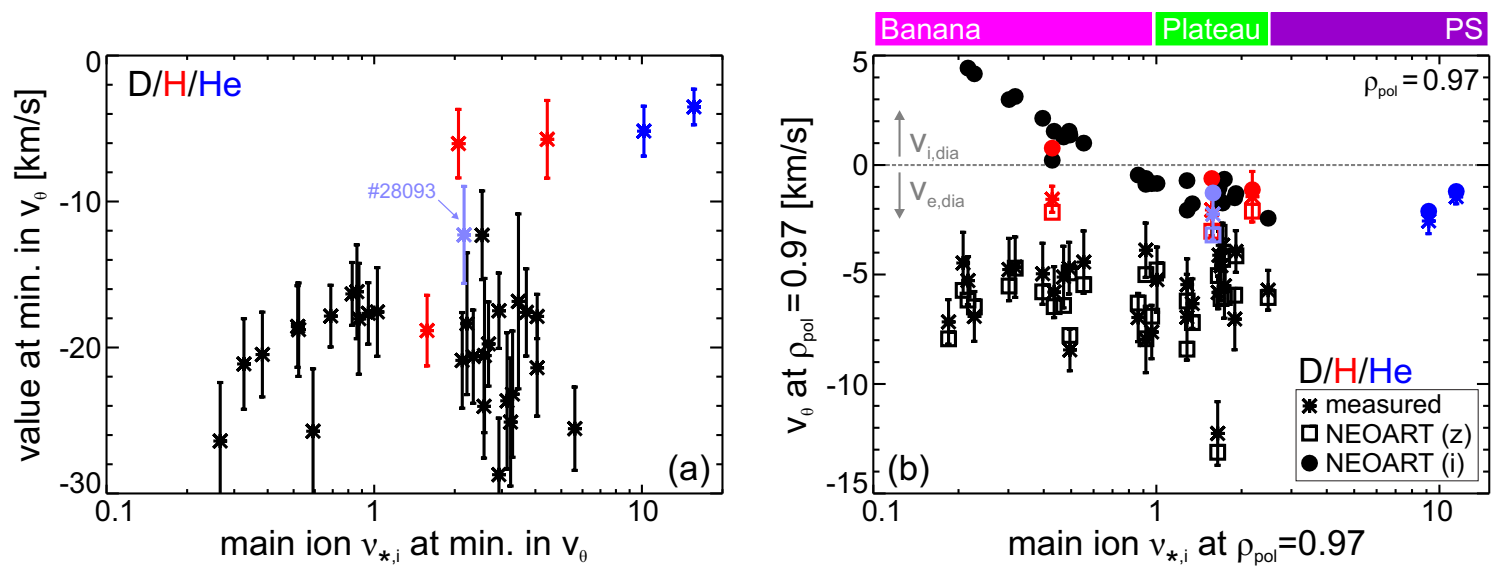

Figure 2: (a) Value at the minimum in the poloidal rotation profile plotted as a function of the main ion collisionality at the same flux surface, (b) comparison between measured and neoclassical poloidal flow at $\rho_{\text {pol }}=0.97$ : Measured $v_{\theta}$ are marked by stars, neoclassical predictions of $v_{\theta, z}$ are highlighted by squares while the neoclassical $v_{\theta, i}$ is marked by circles. Data obtained in deuterium plasmas is shown in black, while hydrogen and helium plasmas are highlighted in red and blue. Note that in helium plasmas the measured poloidal rotation corresponds to the main ion species, obtained via CXRS on $\mathrm{He}^{2+}$, while in deuterium and hydrogen plasmas the measurements are obtained on impurity ions (boron, carbon, nitrogen or neon). 
pedestal $[41,42,27]$.

While $v_{\theta, z}$ does not show a strong dependence on collisionality, $v_{\theta, i}$ scales with this quantity. As shown in figure 2(b), at a given collisionality the neoclassical main ion poloidal rotation flips sign from the electron diamagnetic drift direction $\left(v_{e, d i a}\right)$ to the ion diamagnetic direction $\left(v_{i, d i a}\right)$.

Neoclassical theory also predicts the differential toroidal rotation between main ions and impurities and by invoking the radial force balance equation of both species the main ion toroidal rotation, $v_{\phi, i}$, can be determined from:

$$
\begin{aligned}
v_{\phi, i} & =v_{\phi, z}+\frac{1}{B_{\theta}}\left[\frac{1}{n_{z} q_{z}} \frac{\partial p_{z}}{\partial r}-\frac{1}{n_{i} q_{i}} \frac{\partial p_{i}}{\partial r}+\left(v_{\theta, i}-v_{\theta, z}\right) B_{\phi}\right] \\
& =v_{\phi, z}+\frac{v_{t h, i} \rho_{i}}{2 B_{\theta}} \frac{3 K_{2}}{2} \frac{1}{L_{T_{i}}}
\end{aligned}
$$

with $v_{\phi, z}$ being the impurity toroidal rotation, $B_{\theta}$ and $B_{\phi}$ the poloidal and toroidal magnetic field component, $\frac{\partial p_{z}}{\partial r}$ and $\frac{\partial p_{i}}{\partial r}$ the impurity and ion pressure gradient, $n_{z}$ and $n_{i}$ the impurity and ion density, $q_{z}$ and $q_{i}$ the charge of the impurity and main ion, respectively. $v_{t h, i}$ is the thermal velocity of the main ions, $\rho_{i}$ is the ion Larmor radius, $K_{2}$ is a collisionality-dependent viscosity coefficient as defined in [6] and $L_{T_{i}}$ denotes the ion temperature gradient scale length with $L_{T_{i}}^{-1}=d\left(\ln T_{i}\right) / d r$. Here, we evaluate $v_{\phi, i}$ using the measured impurity flow profiles and the background profiles as well as the neoclassically predicted main ion poloidal flow.

The measured $v_{\phi, z}$ flips sign from counter- to co-current as $\nu_{*, i}$ increases from the banana to the plateau regime. This transition occurs at a similar collisionality to that at which the main ion poloidal flow changes sign in figure 2(b) according to NEOART. Figure 3 shows the minimum in the edge toroidal rotation feature (located typically at the pedestal top position $\rho_{\text {pol }} \approx 0.97$ ) of both impurities and main ions plotted versus $\nu_{*, i}$ at the pedestal top. The impurity toroidal rotation is measured directly, while $v_{\phi, i}$

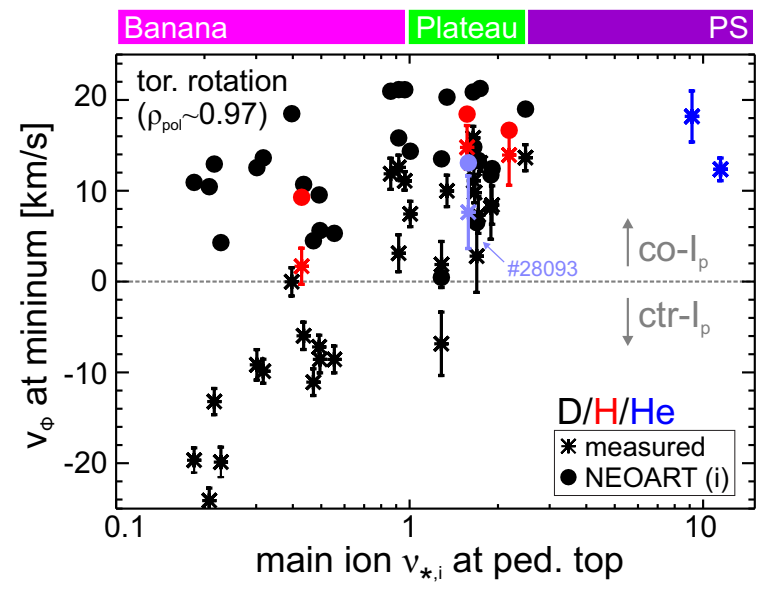

Figure 3: Value at the minimum in the edge toroidal rotation profile $\left(\rho_{\text {pol }} \approx 0.97\right)$ versus main ion pedestal top collisionality: The measurements are marked by stars, while the neoclassical prediction of $v_{\phi, i}$ is highlighted by circles. 
is evaluated from the radial force balance equation. Note that in He plasmas $v_{\phi, i}$ is measured directly. At low collisionality $v_{\phi, z}$ flips sign while the neoclassically predicted $v_{\phi, i}$ remains co-current. The main ion toroidal rotation shows a rather weak (or no) dependence on the main ion collisionality. It should be noted that for deuterium plasmas the viscosity coefficient $K_{2}$ varies from -0.39 (plateau) to -0.85 (banana).

The data suggests that with decreasing collisionality, friction between main ions and impurities becomes less efficient since the differential toroidal rotation between main ions and impurities is predicted to become large and reaches values of up to $30 \mathrm{~km} / \mathrm{s}$. It should be noted that this analysis assumes that $v_{\theta, i}$ can be described by neoclassical theory also in the low collisionality regime. While this has been shown at high collisionality by comparing the experimental data measured in He plasmas to the neoclassical prediction, it remains unclear whether this is true in the low collisionality branch. Recent observations in the plasma core of DIII-D [21] revealed that at very low collisionality the neoclassically predicted differential toroidal rotation between deuterium and impurity ions is larger and even of the wrong sign compared to the measured difference in the toroidal rotation. This discrepancy is explained by an excess of $v_{\theta, i}$ in the ion diamagnetic direction compared to neoclassical values at low collisionality [21]. Further experiments with direct measurements of the main ion poloidal and toroidal flows at the plasma edge are required to elucidate the drive mechanism for the main ion rotation.

\section{In-out impurity asymmetries}

Although transport is often observed to be dominated by anomalous effects, there is growing evidence that under certain conditions the ion transport can be at the neoclassical level. In the pedestal region, where turbulence is strongly reduced, the impurity particle transport [2] has been observed to be near neoclassical values and there is also evidence that the ion heat transport is at the neoclassical level [3]. However, the conventional neoclassical transport can be modified by the existence of poloidal impurity asymmetries; the transport level can be reduced or even increased and the sign of the neoclassical impurity convection can also change depending on the asymmetry level $[43,44]$.

Recent observations show that poloidal impurity asymmetries are present in the plasma core $[45,46,47,48,49,50]$ as well as at the edge $[7,8,9,10]$. Including the physics causing the in-out impurity asymmetry can have an impact on the particle transport level $[49,43]$. The installation of edge CXRS diagnostics at the inboard midplane of AUG, which are comparable to the setup at Alcator C-Mod [51], allowed $E_{r}$ and the impurity kinetic profiles to be studied at two different poloidal locations $[32,8,10]$. This also provides an experimental consistency check of the electrostatic potential $\Phi$ being a flux function, which is discussed below. The comparison of the high-field side (HFS) and low-field side (LFS) profiles requires the alignment of the CXRS measurements and is based on the assumption of constant $T_{i}$ on a flux surface, i.e. the HFS $T_{i}$ profile is shifted 


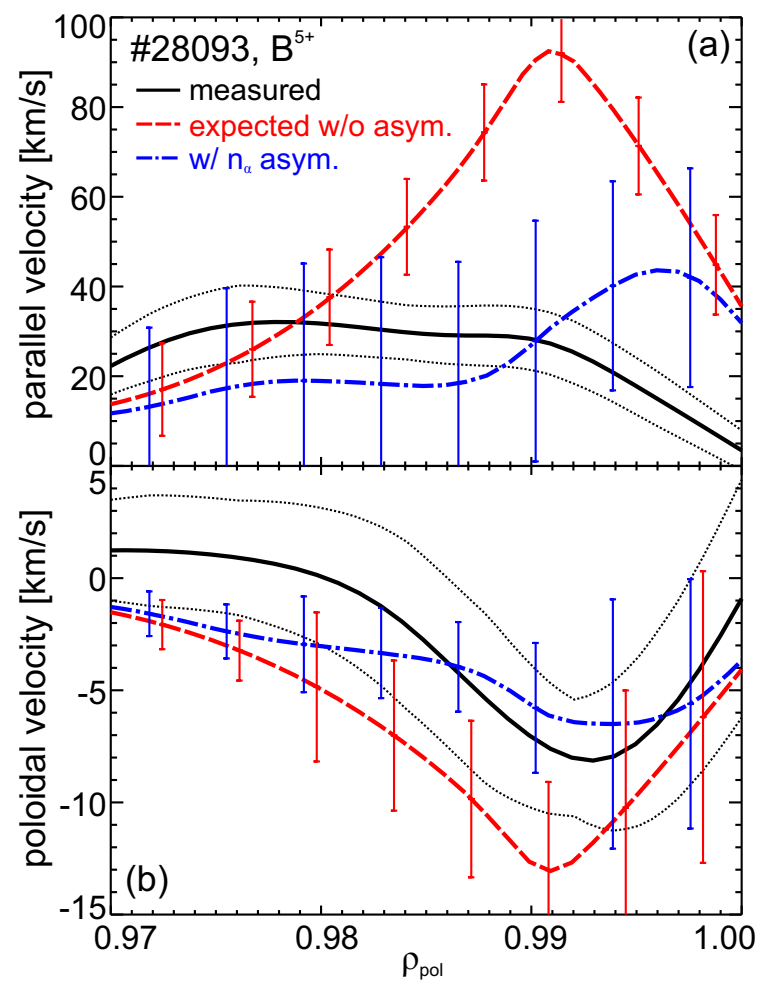

Figure 4: (a) Parallel and (b) poloidal velocities at the HFS: measurement in black (solid lines), profiles computed from LFS flow measurements assuming $n_{z}$ is a flux function in red (dashed lines) and including the $n_{z}$ asymmetry in blue (dashed-dotted lines).

such that it matches the LFS profile. Using this assumption the four independent CXRS measurements at the LFS and HFS revealed the existence of an asymmetric flow pattern along the flux surfaces of the H-mode pedestal $[8,10]$. The flow asymmetry can be explained by an excess of impurity density at the HFS following the postulate of divergence-free flows on a flux surface, which is based on the general continuity equation for a species $z, \nabla \cdot\left(n_{z} \mathbf{v}_{z}\right)=0,[7,8]$. Note that close to the separatrix the validity of $\nabla \cdot\left(n_{z} \mathbf{v}_{z}\right)=0$ may be violated due to the presence of sources or radial transport from neighbouring flux surfaces.

Figure 4 shows the (a) parallel and (b) poloidal velocity of $\mathrm{B}^{5+}$ measured at the HFS (solid black lines) of the H-mode deuterium discharge \#28093 with a toroidal magnetic field on-axis of $-2.5 \mathrm{~T}$, plasma current of $1 \mathrm{MA}, 5 \mathrm{MW}$ of neutral beam injection heating, $1.5 \mathrm{MW}$ of electron cyclotron resonance heating and pedestal top density of $\sim 6 \times 10^{19} \mathrm{~m}^{-3}$. Estimating the effective charge $Z_{\text {eff }}$ from the boron CXRS measurements and including the presence of additional species such as carbon, helium, oxygen and tungsten yields $Z_{\text {eff }} \sim 1.6$ at the pedestal top. The red dashed lines show the HFS parallel and poloidal impurity flows computed from the LFS flow measurements assuming no impurity density asymmetry existed. These profiles are calculated using the divergence-free flow condition $\mathbf{v}_{z}=\omega_{z}(\Psi) R \mathbf{e} \phi+\frac{k_{z}}{n_{z}} B \mathbf{e}_{\|}$and assuming that $n_{z}$ is constant along the flux surfaces. The error bars shown in figure 4 are evaluated by 
means of Gaussian error propagation using the standard deviation of the measured data in a small radial interval, i.e. \pm 0.005 in $\rho_{\text {pol }}$. The predicted velocities are up to a factor of $\sim 4(\sim 2)$ too high for the parallel (poloidal) flow, indicating the presence of a poloidally asymmetric $n_{z}$. Note that for the poloidal flow the uncertainties are too large and within the error bars the measured profile may also be described with a constant $n_{z}$ profile. The LFS impurity density profile is derived from the intensity of the active CX line measured with the LFS diagnostics [10]. Effects due to the beam halo, which is produced by charge exchange between beam neutrals and deuterium ions, are included. The HFS $n_{z}$ profile can be evaluated from the measured charge exchange radiance if the neutral density is known. We obtain the neutral density using two different methods, detailed in [10]. One method employs the measured $\mathrm{D}_{\alpha}$ signal, and the other method uses modelling of the gas puff penetration using the code KN1D [52]. Using both evaluation methods the density is up to a factor of 3 higher at the HFS than at the LFS, demonstrating that in the edge pedestal $n_{z}$ is asymmetric on a flux surface [10]. The radial $n_{z}$ profiles at the LFS and HFS for the discharge of figure 4 are shown in figure 5 of ref. [10]. The asymmetry in $n_{z}$ is in agreement with measurements at Alcator C-Mod $[9,53]$ which demonstrated in-out impurity density asymmetries of up to a factor of $\geq 10$. Accounting for the measured poloidal asymmetry in the $\mathrm{B}^{5+}$ density (see figure 8 ), the HFS and LFS measurements are consistent with the condition of divergence-free flows (see blue lines in figure 4). Note that the absolute shape of the predicted profile does not exactly match the measured profile. This might be explained by the radial uncertainties, since small errors in the radial alignment that are below the accuracy $(2-3 \mathrm{~mm})$ can have an impact on the absolute shape.

By comparing the $E_{r}$ measurements from the HFS and LFS, we can compute how much the electrostatic potential $\Phi$ would have to vary on a flux surface for the assumed LFSHFS relative alignment. At Alcator C-Mod, recent results showed that the electrostatic potential and impurity temperature are not concurrently constant on flux surfaces [54]. Assuming that $T_{i}$ is a flux function results in large in-out asymmetries of $\Phi$ and the electron density at C-Mod [54]. Using the assumption of a constant plasma potential, i.e. aligning the radial locations of the $E_{r}$ well between the HFS and LFS, the C-Mod measurements imply a poloidal variation of the impurity temperature, with the impurity temperature being higher at the LFS than at the HFS [54].

At AUG, the radial position of the minimum in the poloidal rotation profile matches quite well between the HFS and LFS when assuming that $T_{i}$ is a flux function. Since in $\mathrm{H}$-mode the poloidal rotation term of the impurity force balance is the dominant contribution to the evaluation of $E_{r}[42,37]$, this suggests that the electrostatic potential is constant on the flux surfaces. Figure $5(\mathrm{a})$ shows the $T_{i}$ profile measured at the LFS in black and at the HFS in red. Note that here we use $T_{i} \approx T_{z}$, with $T_{z}$ being the measured impurity ion temperature, since the equilibration time between main ions and impurities is short compared to local transport time scales [37]. The $E_{r}$ profiles are shown in figure $5(\mathrm{~b})$. Note that the radial locations of the $E_{r}$ wells align reasonably well. The difference in the radial position is $\sim 1 \mathrm{~mm}$, which is below the uncertainty of 


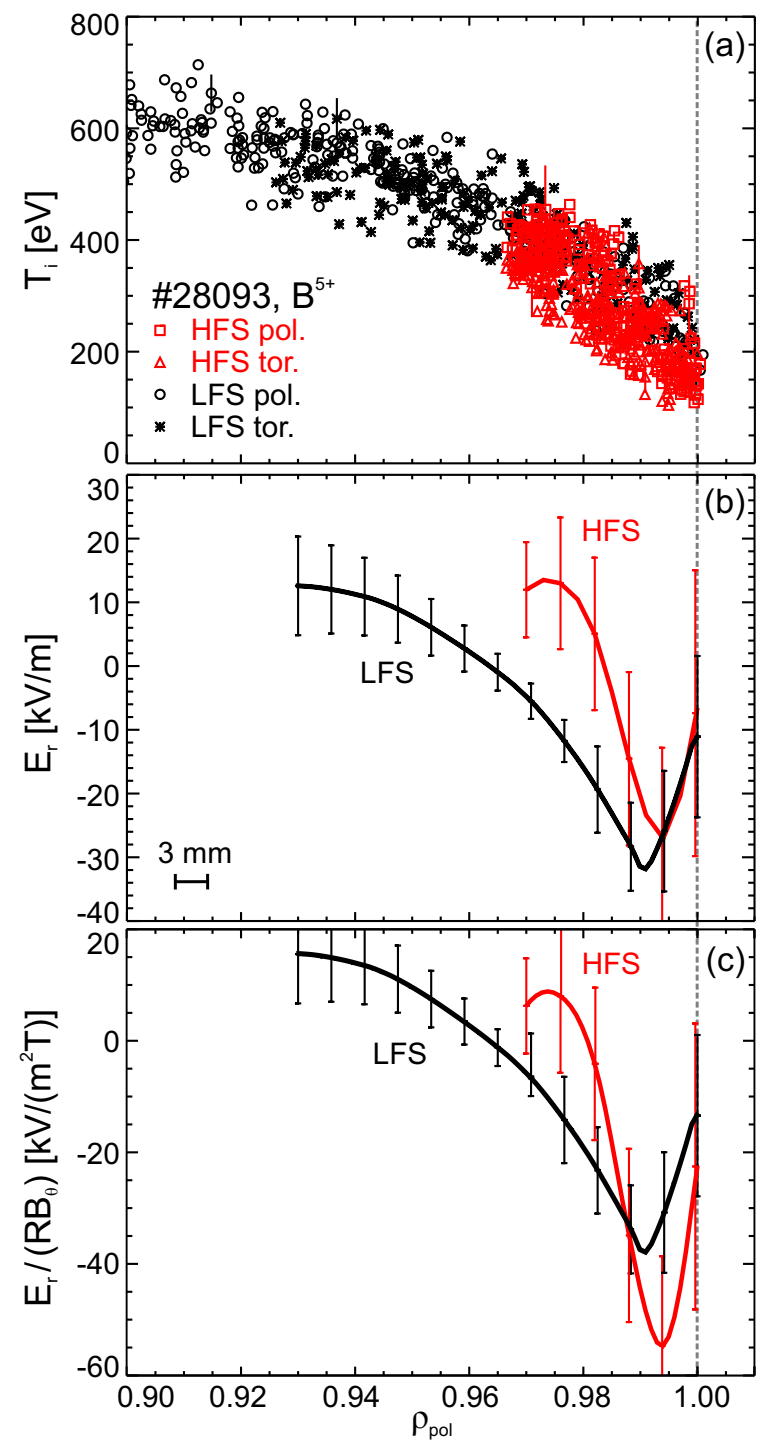

Figure 5: Measurements of HFS (red) and LFS (black) profiles: (a) $T_{i}$, (b) $E_{r}$ and (c) $E_{r} /\left(R B_{\theta}\right) \propto \partial \Phi / \partial \Psi$.

the relative profile alignment $(2-3 \mathrm{~mm})$. Figure $5(\mathrm{c})$ shows the radial HFS and LFS profiles of $E_{r} /\left(R B_{\theta}\right)$ which is proportional to $\frac{\partial \Phi}{\partial r}, \Phi$ being the electrostatic potential. Within the radial uncertainties the profiles are consistent with the assumption that $\Phi$ is a flux function (see also figure 6).

The electrostatic potential $\Phi$ can be derived from the $E_{r}$ measurements using $E_{r}=-\nabla \phi$ and is obtained by integrating $E_{r}$ along the radial coordinate $r$. For the calculation of $\Phi$ we assumed that the electrostatic potential is a flux function inside of the edge transport barrier, i.e. $\Phi_{\text {ped,top }}^{\mathrm{LFS}}=\Phi_{\text {ped,top }}^{\mathrm{HFS}}=\Phi_{0}$ is set as a starting condition at the pedestal top, where $\Phi_{0}$ is chosen arbitrarily. Figure 6 shows the electrostatic potential at the HFS and LFS. Evaluating the electric potential profiles from the measured $E_{r}$ profiles indicates that $\Phi$ may be lower at the HFS compared to the LFS, however, taking the experimental uncertainties into account, $\Phi$ could also be a flux function, forming a 


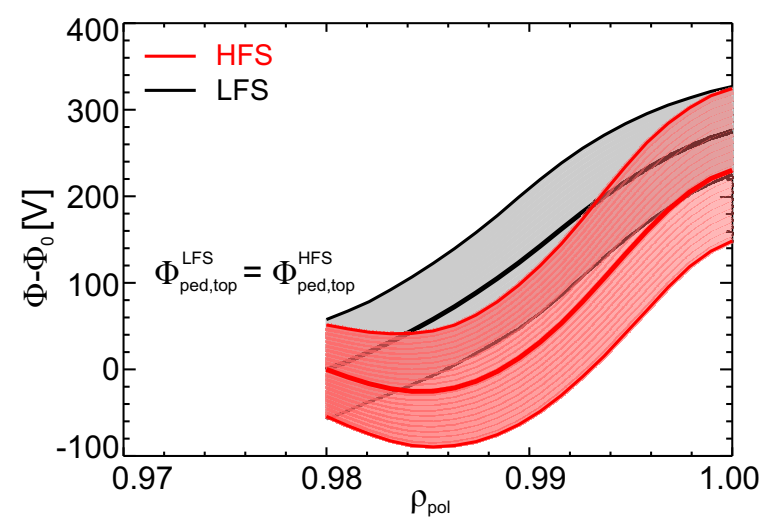

Figure 6: Evaluation of the electrostatic potential at the LFS (black) and HFS (red).

consistent equilibrium with the measured flow structure.

The in-out $n_{z}$ asymmetry was measured in a range of $\mathrm{H}$-mode plasmas with different impurity species (here, $\mathrm{B}^{5+}$ and $\mathrm{N}^{7+}$ ) and different collisionalities. The in-out asymmetries in the pedestal are present for both boron and nitrogen, with an impurity accumulation at the HFS, reaching asymmetry factors, $n_{z}^{\mathrm{HFS}} / n_{z}^{\mathrm{LFS}}$, of $\sim 2.5-3.5$. At low collisionality, when a shift of the LFS edge $v_{\phi, z}$ into the counter-current direction is observed, also the HFS profile is shifted, eventually changing sign and becoming counter-current close to the separatrix. A fluid model which will be discussed in section 4.1 predicts a collisionality dependence of the impurity density asymmetry factor. This can also be seen in the experimental data (see figure 7), however, the error bars on the measurement are too large to be able to say anything definitive and a statistical number of measurements is not available at this time. In figure 7 the maximum in-out impurity asymmetry is plotted as a function of the main ion collisionality at the flux surface,

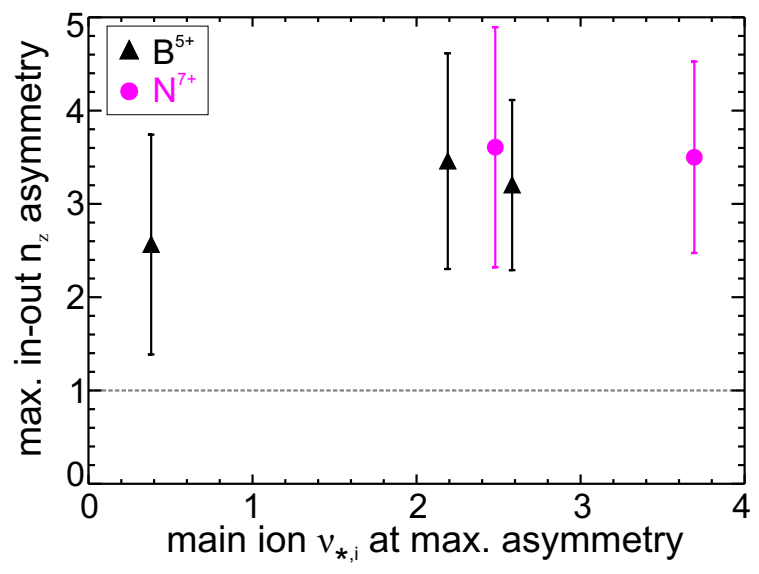

Figure 7: Maximum impurity density asymmetry factor, defined as the ratio between the measured impurity density at the HFS and the LFS, plotted as a function of the main ion collisionality at the location of the maximum asymmetry: data measured on $\mathrm{B}^{5+}$ is shown in black, while $\mathrm{N}^{7+}$ measurements are shown in magenta. 
where the impurity asymmetry factor has its maximum.

\subsection{Consistency with parallel momentum balance}

As we observe poloidally asymmetric impurity densities, we present the predictions of a fluid model which describes this in-out impurity variation in combination with the results of numerical simulations with a drift-kinetic code. The details of the fluid model are given in the following subsection while the comparison between modelling and experiment is presented in section 4.1.2.

4.1.1. In-out impurity variation on a flux surface from a fluid model Collisional processes determine the equilibration of flows and particle densities on a flux surface. Using 'classical' parallel momentum balance, the shape of the density and the flow profile on a flux surface can be reconstructed. 'Classical' means that at lowest order the orbit width effects are neglected (which can be justified in high-collisionality regimes). Previous work assumed dominant friction between impurities and main ions [55] as well as rigid toroidal rotation of the bulk plasma $[56,57]$. The inertial term in the parallel momentum balance of impurities has also been considered as a possible player $[58,44]$. In particular, the inertial term related to the poloidal impurity flow plays an important role if the poloidal Mach number, defined as $M_{\text {pol }}=\left(v_{\theta} B\right) /\left(v_{t h} B_{\theta}\right)$, approaches unity. For the main ions, this could lead to the appearance of static shock fronts since the magnetic field acts effectively like a nozzle [59]. For a trace impurity, this is prohibited by the linearity of the system, i.e. a shock can only be a transient phenomenon. Nevertheless, the poloidal centrifugal force becomes substantial and of the same order as the 'toroidal' centrifugal force related to the $\mathbf{E} \times \mathbf{B}$ flow (thus keeping the total parallel/toroidal centrifugal forces small). As such, inclusion of the poloidal centrifugal force as well as the centrifugal force related to the rigid toroidal rotation is important as they actually almost balance each other [60].

In the following we introduce the main equations and assumptions of the fluid model that has been used to study the measured in-out impurity variations. Using the particle continuity equation at stationarity, $\nabla \cdot\left(n_{z} \mathbf{v}_{z}\right)=0$, the poloidal dependence of the poloidal flow component can be derived:

$$
v_{\theta, z}=k_{z}(\Psi) \frac{B_{\theta}}{n_{z}}
$$

where $k_{z}(\Psi)$ is a flux function. Here, it is assumed that the divergence of the radial flow is negligible. Using the general velocity expressions $\mathbf{v}=v_{\|} \mathbf{b}+v_{\perp}$ and $\mathbf{v}=v_{\phi} \mathbf{e}_{\phi}+v_{\theta} \mathbf{e}_{\theta}$, the parallel component of the flow can be written as:

$$
\mathbf{v}_{z} \cdot \mathbf{e}_{\|}=v_{\|, z}=\omega_{z} R \frac{B_{\phi}}{B}+k_{z}(\Psi) \frac{B}{n_{z}}
$$

where $\omega_{z}$ is the rigid rotation frequency. From these basic considerations the flux function $k_{z}$ can be determined via $k_{z}(\Psi)=\frac{\left\langle B v_{\|, z}\right\rangle}{\left\langle B^{2} / n_{z}\right\rangle}-\omega_{z} R \frac{B_{\phi}}{\left\langle B^{2} / n_{z}\right\rangle}$, where $\langle$.$\rangle denotes$ the flux-surface average. A pure toroidal flow can be sustained on a flux surface by 
choosing $\left\langle B v_{\|, z}\right\rangle=\omega_{z} R B_{\phi}$, while a poloidal flow is always accompanied by a toroidal flow which exhibits in-out asymmetries (Pfirsch-Schlüter flow) [4].

The parallel force balance equation for a species $z$ considered here includes friction, the thermal force, the differential toroidal rotation term, the centrifugal force, the pressure force, the stress tensor (anisotropy) and the electric force (from left to right):

$$
\begin{aligned}
& -\nu_{z i} \mathbf{B} \cdot\left(\left(\mathbf{v}_{z, \|}-\mathbf{v}_{i, \|}\right)+\Delta Q_{\|} \cdot \frac{\mathbf{B}}{B}+\delta v_{\|} \frac{\mathbf{B}}{\left\langle B^{2}\right\rangle}\right)= \\
& \mathbf{B} \cdot \nabla\left(\frac{v_{\theta, z}^{2} B^{2}}{2 B_{\theta}^{2}}-\frac{R^{2} \omega_{z}^{2}}{2}\right)+\mathbf{B} \cdot \frac{\nabla P_{\|}}{n_{z}}+\left(T_{\perp}-T_{\|}\right) \mathbf{B} \cdot \nabla \log (B)+Z_{z} \mathbf{B} \cdot \nabla \tilde{\Phi}
\end{aligned}
$$

The friction force, with $\nu_{z i}$ being the collisional frequency between impurity and main ion species, leads to inboard accumulation $[44,55]$, the thermal force causes an updown asymmetry and thus, acts to reduce the in-out asymmetry. The thermal force is expressed here as: $\Delta Q_{\|}=C \frac{1}{e B} \frac{B_{\phi}}{B_{\theta}}\left(\frac{B^{2}}{\left\langle B^{2}\right\rangle}-1\right) \frac{d T_{i}}{d r}$ [55] with a constant $C$, which is used to scan the thermal force contribution (see below). Note that the expression for the thermal force [55] is derived for the high collisionality regime and is applied outside its domain of validity, but serves to estimate its contribution to the in-out impurity asymmetry. $\delta v_{\|}$is the differential toroidal rotation and is set to 0 in the presented simulations. The centrifugal force can lead to both an inboard or an outboard accumulation. The toroidal centrifugal force associated with the rigid toroidal rotation $\left(\propto \frac{R^{2} \omega_{z}^{2}}{2}\right)$ tends to accumulate the impurities at the LFS, while the poloidal centrifugal force $\left(\propto \frac{v_{\theta, z}^{2} B^{2}}{2 B_{\theta}^{2}}\right)$ leads to an inboard impurity localization. The pressure force $\left(\propto \frac{\nabla P_{\|}}{n_{z}}\right.$, where $P_{\|}=n_{z} T_{\|}$is the pressure in the parallel direction) causes a re-equilibration and works against an asymmetric situation. The anisotropic term $\left(\propto\left(T_{\perp}-T_{\|}\right)\right)$ causes poloidal damping while the electric force leads to an inboard or an outboard accumulation depending on the sign of $\nabla \tilde{\Phi}$. Note that $T_{\perp}$ and $T_{\|}$are solved using higher order moments of the drift kinetic equation (see e.g. [56]).

The solved equations are obtained from moments of the steady-state drift-kinetic equation for the impurity equilibrium distribution function $f$ (in $v_{\|}, \mu$ velocity coordinates):

$$
\nabla \cdot\left[\left(v_{\|} \mathbf{b}+\mathbf{v}_{\mathrm{E}}\right) B f\right]+\frac{\partial}{\partial v_{\|}}\left(\dot{v}_{\|} B f\right)=B C
$$

where $\mathbf{v}_{\mathrm{E}}=\mathbf{E} \times \mathbf{B} / B^{2}, \mathbf{E}=-\nabla \Phi_{0}$, and $C$ is the impurity-ion collision operator. All terms inversely proportional to the charge $Z$ have been neglected (i.e. the curvature and centrifugal drifts). The parallel acceleration is given by: $\dot{v}_{\|}=-\mu \mathbf{b} \cdot \nabla B-Z e / M \mathbf{b} \cdot \nabla \tilde{\Phi}+$ $v_{\|} \frac{\mathbf{v}_{\mathrm{E}} \cdot \nabla B}{B}+\mathbf{v}_{\mathrm{E}} \cdot\left[\left(\mathbf{v}_{\mathrm{E}} \cdot \nabla\right) \mathbf{b}\right]$, where it is important to retain both terms involving $\mathbf{v}_{\mathrm{E}}$. The distribution function $f$ is assumed to be a shifted bi-Maxwellian with higher-order corrections:

$$
f=\frac{n}{(2 \pi)^{3 / 2} \sqrt{T_{\|} T_{\perp}^{2}}} e^{-0.5\left(v_{\|}-U_{\|}\right)^{2} / T_{\|}-\mu B / T_{\perp}}\left(1+\left(v_{\|}-U_{\|}\right) C_{q K}\right)
$$


$C_{q K}=\left[\frac{\left(v_{\|}-U_{\|}\right)^{2}}{2 T_{\|}}-\frac{3}{2}\right] \frac{q_{\|}}{n T_{\|}^{2}}+\left(\mu B / T_{\perp}-1\right) \frac{K_{\|}}{n T_{\|} T_{\perp}}$

where $n, U_{\|}, T_{\|}, T_{\perp}, q_{\|}, K_{\|}$are the fluid moments unknowns to be solved for. This formulation is suited when $U_{\|} \sim v_{\text {th }}$ but $q_{\|}, K_{\|} \ll n v_{\text {th }}$. Note that the mass $M$ has been fixed to unity in appropriate units. Plugging (7) into (6) and taking the moments integrals of equation (7), one gets, after some algebra and the use of the relevant identity $\mathbf{v}_{\mathrm{E}} \cdot\left[\left(\mathbf{v}_{\mathrm{E}} \cdot \nabla\right) \mathbf{b}\right]=\mathbf{b} \cdot \nabla\left[\frac{R^{2} \Omega^{2}}{2} \frac{B_{\theta}^{2}}{B^{2}}\right]$, the system of equations:

$\mathbf{B} \cdot \nabla\left(n \frac{\hat{u}}{B}\right)=0$

$\nabla_{\|} T_{\|}+T_{\|} \nabla_{\|}(\log n+Z \tilde{\Phi})+\left(T_{\perp}-T_{\|}\right) g_{\mathrm{B}}+\nabla_{\|}\left(\frac{\hat{u}^{2}}{2}-\frac{R^{2} \Omega^{2}}{2}\right)=$

$$
=-\sigma\left(U_{\|}-U_{\mathrm{i}}+\Delta Q_{\|}\right)
$$

$\frac{B}{n} \mathbf{B} \cdot \nabla\left(\frac{K_{\|}}{B^{2}}\right)+\hat{u} \mathbf{B} \cdot \nabla\left(\frac{T_{\perp}}{B}\right)=-\sigma\left(T_{\perp}-1\right)$

$\frac{\mathbf{B}}{n} \cdot \nabla\left(\frac{3 q_{\|}}{B}\right)+\hat{u} \nabla_{\|} T_{\|}+2 T_{\|} \nabla_{\|} \hat{u}+2 \frac{K_{\|}}{n} g_{\mathrm{B}}=$

$$
=-\sigma\left(T_{\|}-1\right)+\sigma\left(U_{\|}-U_{\mathrm{i}}\right)^{2}
$$

$B \mathbf{B} \cdot \nabla\left(\frac{K_{\|} \hat{u}}{B^{2}}\right)+K_{\|} \nabla_{\|} \hat{u}+n T_{\|} \mathbf{B} \cdot \nabla\left(\frac{T_{\perp}}{B}\right)+n T_{\perp}^{2} g_{\mathrm{B}}=$

$$
=-\sigma n\left(U_{\|}-U_{\mathrm{i}}\right)\left(1-T_{\perp}\right)-\sigma K_{\|}
$$

$\mathbf{B} \cdot \nabla\left(\frac{q_{\|} \hat{u}}{B}\right)+n T_{\|} \nabla_{\|} T_{\|}+3 q_{\|} \nabla_{\|} \hat{u}=$

$$
=-\sigma q_{\|}+\sigma n\left(U_{\|}-U_{\mathrm{i}}\right)\left(T_{\|}-1\right)-\frac{\sigma n}{3}\left(U_{\|}-U_{\mathrm{i}}\right)^{3}
$$

where $\Omega=\omega_{\mathrm{z}}=-d \Phi / d \Psi, U_{\|}=\mathbf{v}_{z} \cdot \mathbf{e}_{\|}, \hat{u}=v_{\theta, z} B / B_{\theta}$, and the terms proportional to $\sigma$ come from the collision operator, assuming a simple Krook-type operator, with $\sigma$ the impurity-ion collisional frequency. As in equation (5), here the heat flow friction term $\Delta Q_{\|}$is added as an ad-hoc term. Also $g_{\mathrm{B}}=\nabla_{\|} \log B$. Note that the second equation of the system (8) is one and the same equation (5). The poloidally varying potential perturbation $\tilde{\Phi}$ is assumed to be small compared to the dominant background equilibrium potential $\Phi_{0}$ and is set to 0 in the simulations discussed below. A-posteriori this is justified by checking that the parallel pressure gradient is larger than the parallel electric force due to either the centrifugal potential or the Pfirsch-Schlüter potential. This is also consistent with the finding that the electric potential is a flux function inside the error bars of the electric field determination at the LFS and HFS.

The parallel momentum balance, together with the higher order moments, is solved numerically for $n_{z}$ and the flows are then connected to the particle density via the divergence-free flow condition. For the evaluation of $\mathbf{v}_{i, \|}$ via equation (5) the following approach has been employed:

(i) The measured LFS impurity toroidal and poloidal rotation profiles are used to 
derive $\Omega$ that goes into equation (8), as $\Omega=\frac{1}{R^{L F S}}\left(v_{\phi, z}^{L F S}-v_{\theta, z}^{L F S} \frac{B_{\phi}^{L F S}}{B_{\theta}^{L F S}}\right)$.

(ii) Given the LFS measured impurity density and the simulated profile, the main ion poloidal rotation is computed as: $\frac{n_{i} v_{\theta, i}}{B_{\theta}}=\left(\frac{n_{\mathrm{z}}^{L F S} v_{\theta, z}^{L F S}}{B_{\theta}^{L F S}}\left\langle\frac{B^{2}}{n_{z}}\right\rangle+R B_{\phi}\left(\Omega-\omega_{\mathrm{i}}\right)\right) /\left\langle B^{2}\right\rangle$.

(iii) The unkown $\omega_{\mathrm{i}}$ is adjusted to get a good match of the HFS impurity toroidal rotation profile in the pedestal region. A-posteriori, it is checked that $\omega_{i}$ falls inside its experimental error bars determined from radial force balance applied to the main ions, where the poloidal velocity is taken from NEOART. Similarly, the poloidal velocity computed from the previous item is also compared to NEOART.

In the following the analytical approach of this fluid model is applied to the experimental data.

4.1.2. Comparison between experiment and theory The fluid model can reproduce the measured impurity flows and the asymmetry factor when a finite poloidal flow of the main ions is included in the model [10]. In figure 8 the asymmetry factor predicted by the fluid model is compared to the measurement. The black curve is evaluated from the measurement of the $\mathrm{B}^{5+}$ and $\mathrm{D}_{\alpha}$ spectral line while for the blue curve KN1D has been used to simulate the penetration of the gas cloud. The red and green curve (labelled as case 1 and case 2) correspond to two simulations, in which the thermal force contribution has been scanned (see below). As can be seen in figure 8(a) the level of in-out asymmetry can be reproduced by the fluid model. Figure 8 (b) shows the poloidal dependence of the boron density profile along the flux surfaces.

We have studied a possible collisionality dependence of the impurity asymmetry factor using the fluid model. Here the experimental data for the $\mathrm{B}^{5+}$ discharge (\#28093) was used and the nominal collision frequency was scanned. Figure 9 shows the in-out impurity asymmetry factor as a function of the ion collisionality. For the first version of the fluid model [10], where the viscous stress tensor was neglected, the simulations show a rather weak collisionality dependence of the impurity asymmetry factor (see black triangles in figure 9$)$. Including the viscosity term $\left(\propto\left(T_{\perp}-T_{\|}\right)\right.$in equation $\left.(5)\right)$ results in similar asymmetry factors at higher collisionality, while in low collisional plasmas the viscous stress tensor becomes important and reduces the in-out asymmetry (see red squares in figure 9). This indicates that the impurity density asymmetry scales with the ion collisionality, with smaller asymmetry factors at low collisionality.

Analysis of the different contributions to the parallel momentum balance reveals the nature of the impurity dynamics. The simulations show that the impurity asymmetry develops due to the interplay of all forces. Figure 10 shows a comparison of the forces resulting from the fluid treatment (dashed lines) and from the kinetic code HAGIS (solid lines). In HAGIS the evolution of the deviation $\delta f$ is obtained from the full variation of the Maxwellian $f_{0}$ and the collisions with the main ions. The collision operator is linear in $\delta f, f_{0}$ does not contribute, since the mean velocity is the main ion 

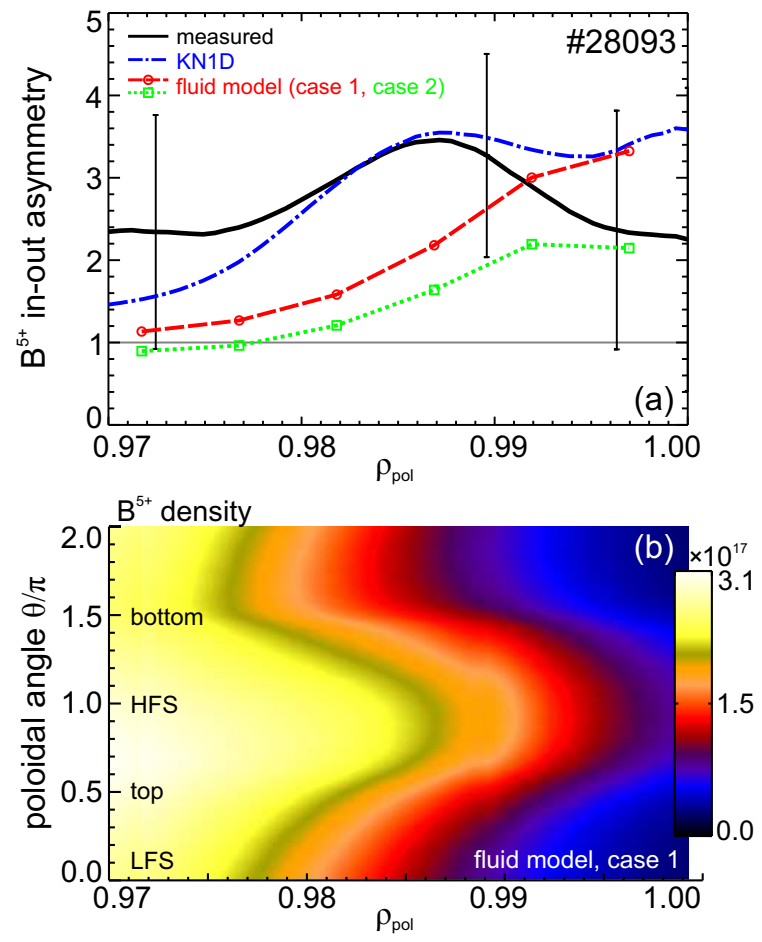

Figure 8: (a) Asymmetry factor of boron as derived from the measurement using the $\mathrm{B}^{5+}$ and the $\mathrm{D}_{\alpha}$ spectral line in black, using KN1D in blue and simulated by the fluid model in red and green, (b) poloidal $\mathrm{B}^{5+}$ density distribution predicted by the fluid model.

velocity. Therefore $\left|\delta f / f_{0}\right| \ll 1$ is not required. The simulations with HAGIS were performed for a trace impurity in two steps. First, a calculation for the deuterium ions neglecting the impurities, then for the impurities with collisions with deuterium. The

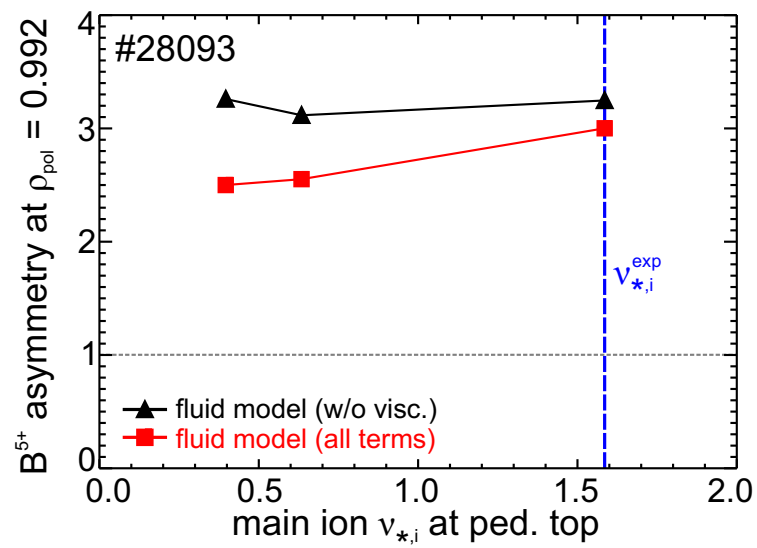

Figure 9: Collisionality scaling of in-out impurity asymmetries: with lower collisionalities the fluid model predicts smaller asymmetry factors, as observed in experiment. Including the viscous stress tensor (red squares) shows a stronger collisionality dependence. The blue dashed line marks the experimental ion collisionality. 


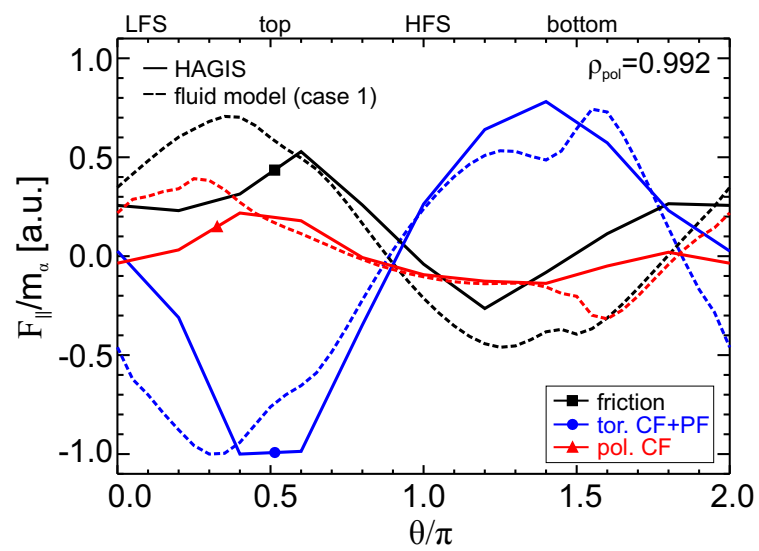

Figure 10: Comparison between fluid model (dashed lines) and kinetic simulations using HAGIS (solid lines): the friction force is shown in black, the sum of the toroidal centrifugal force (tor. $\mathrm{CF}$ ) and the pressure force $(\mathrm{PF})$ in blue, and the poloidal centrifugal force (pol. CF) in red.

simulations determine $\delta f$ from $f_{0}$ which is chosen to have the same average velocity and temperature as the deuterium ions. The range $\rho_{\text {pol }}$ from 0.8 to 0.999 is covered and the marker particles are reflected at the boundaries. In the simulations the ratio between inboard and outboard density is for most flux surfaces lower than two, the deviation from the average is up to about $50 \%$. In figure 10 the friction force is shown in black, the poloidal centrifugal force in red and the sum of the toroidal centrifugal force and the pressure drive in blue. The relative behaviour of the different terms along the poloidal arc shows good agreement between the fluid and kinetic approach.

Using the fluid model the contributions of the individual terms in the parallel momentum balance can be studied. Figure 11 shows the different terms for two radial positions, (a) $\rho_{\text {pol }}=0.98$ and (b) $\rho_{\text {pol }}=0.992$. At both locations the friction force (black squares) is to a large extent balanced by the $\nabla n$ part of the pressure drive (magenta stars), while the other forces give smaller contributions. Further inwards $\left(\rho_{\text {pol }}=0.98\right)$ the poloidal centrifugal force (red circles) is small and plays only a minor role, while towards the separatrix $\left(\rho_{p o l}=0.992\right)$, where $v_{\theta, z}$ approaches its maximum, the fraction increases (see figure 11(b)). This shows the importance of the poloidal centrifugal term for the impurities, which is usually neglected.

Recent B2SOLPS5.2 simulations including all drift and current terms [60] reproduced the $\mathrm{B}^{5+}$ impurity asymmetry qualitatively. An in-out impurity asymmetry of $\sim 2$ was obtained in the simulations which further corroborate that in the presence of strong temperature and density gradients neoclassical effects lead to such asymmetries [61]. For the flux surface located $1 \mathrm{~cm}$ inside the separatrix (roughly at $\rho_{\text {pol }}=0.98$ ) the simulations show that friction and the thermal force are dominant [60].

To study the impact of the thermal force we have increased its contribution in the parallel momentum balance using the fluid model. Compared to the simulations shown in figure 11 (fluid model, case 1) we have multiplied the constant $C$ in the thermal force 


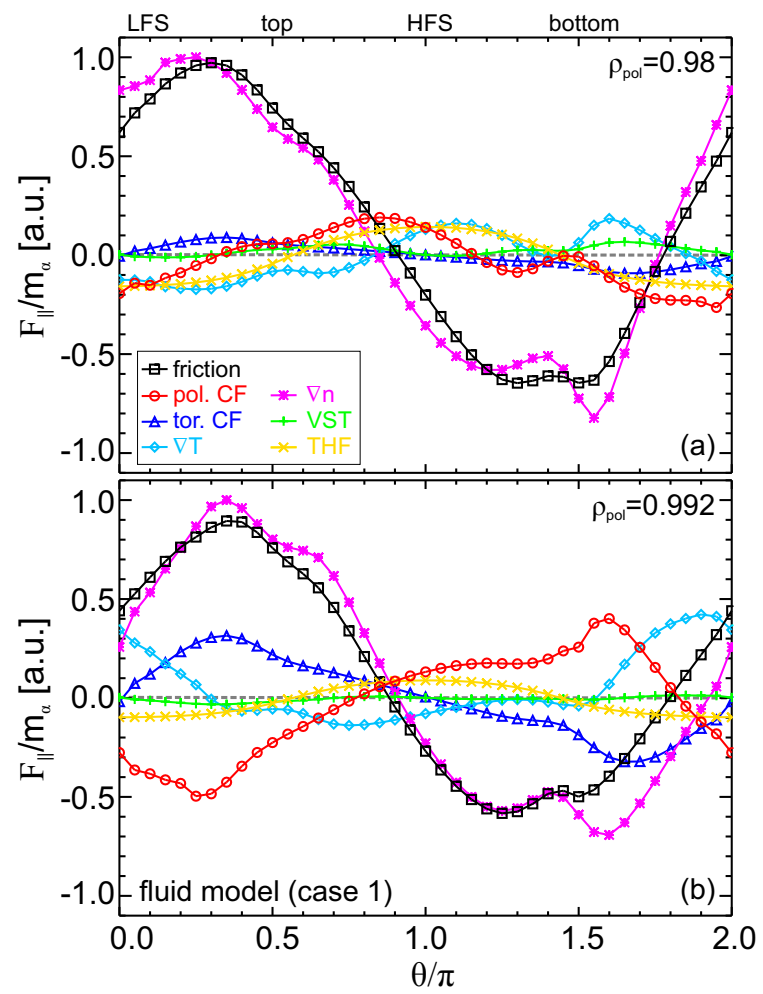

Figure 11: Relative contributions of the different terms in the parallel force balance at two radial locations $\left((\mathrm{a}) \rho_{\text {pol }}=0.98\right.$ and (b) $\left.\rho_{\text {pol }}=0.992\right)$ : the friction force is shown in black (squares), the poloidal centrifugal force (pol. CF) in red (circles), the toroidal centrifugal force (tor. CF) in blue (triangles), the force due to the temperature gradient $(\nabla T)$ and density gradient $(\nabla n)$ in light blue (diamonds) and magenta (stars), the viscous stress tensor (VST) contribution in green (crosses) and the thermal force (THF) in yellow (diagonal crosses).

contribution by a factor of 3 . This may be justified since (i) $\nabla T_{i}$, which enters in the thermal force, has experimental uncertainties and (ii) $C$ depends on the collisionality regime. Figure 12 shows the resulting relative contributions of the different terms in the parallel momentum balance at the same radial positions, (a) $\rho_{\text {pol }}=0.98$ and (b) $\rho_{\text {pol }}=0.992$. The friction force is now mainly balanced by the thermal force, while the pressure drive ( $\nabla n$ and $\nabla T$ contribution in magenta and light blue) is now significantly smaller compared to case 1 . Note, however, that in the edge pedestal the centrifugal force still plays an important role for driving the asymmetries, with a larger contribution close to the separatrix at $\rho_{\text {pol }}=0.992$. Increasing the thermal force also has an impact on the flow structure and the impurity density distribution along the flux surfaces. Since the thermal force causes an up-down asymmetry and tends to decrease an in-out impurity asymmetry, the asymmetry factor shown in figure 8 (green curve, labelled as 'fluid model, case 2') is also reduced.

In summary, the comparative analysis between modelling and experiments presented here show that in the edge transport barrier the impurity density distribution is not a 


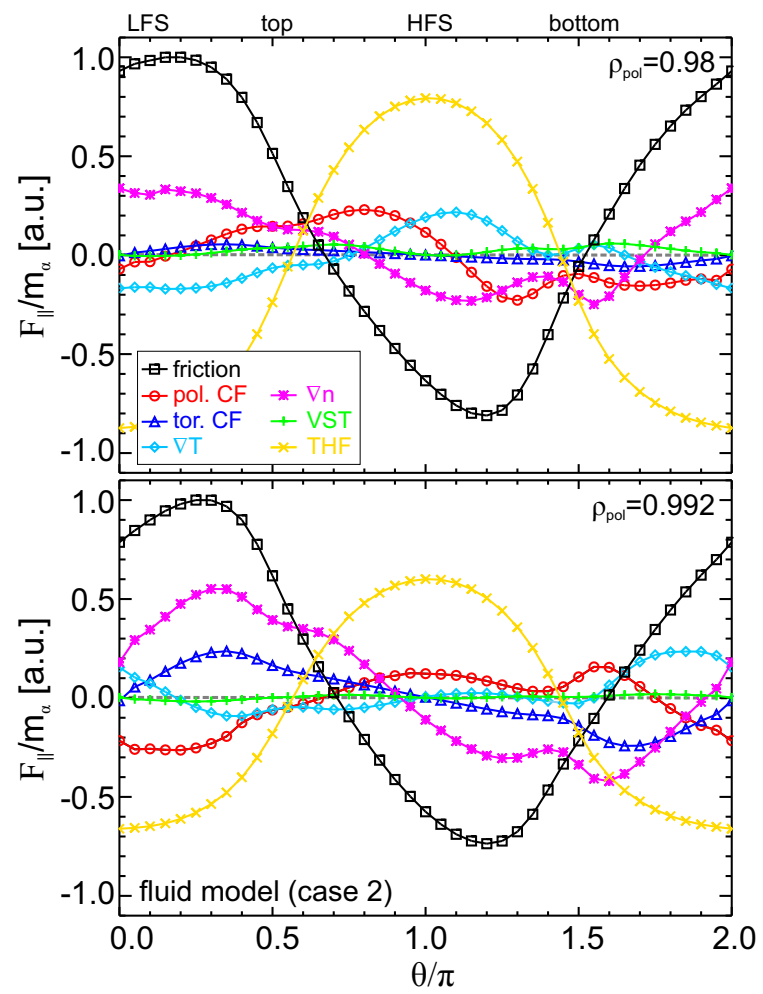

Figure 12: Simulation for an increased thermal force contribution (multiplied by a factor of 3 compared to case 1 shown in figure 11). The labelling of the different terms in the parallel force balance is the same as in figure 11.

flux function and accumulates at the HFS. The simulations show that the asymmetries in the impurity density and flow profiles are caused by the interaction between all forces in the parallel momentum balance. Modelling using the fluid model and the kinetic code shows a consistent picture. In the plasma edge, friction between main ions and impurities is dominant. The inertial term emerging from the poloidal impurity flow can become important, especially in the region where the poloidal Mach number approaches unity, i.e. close to the separatrix. The fact that the impurity density has a poloidal dependence has an impact on the total flow on a flux surface and is responsible for the features of both the toroidal (parallel) and poloidal flows, as observed in experiment.

\subsection{Neoclassical impurity transport with and without poloidal asymmetries}

Due to a re-equilibration of the impurities on the flux surfaces, the neoclassical particle flux has a non-linear dependence on the gradients in density and temperature [55]. The in-out impurity density asymmetry affects the flow structure on the flux surfaces and has an impact on the Pfirsch-Schlüter transport [43]. The neoclassical fluxes are changed and the $v$ and $D$ transport coefficients, with $v$ being the convective velocity and $D$ the diffusion coefficient, are affected. The in-out impurity asymmetry tends to reduce $v$ and $D$ to smaller values. Figure 13 shows the impurity transport coefficients and the impurity flux normalized to the particle density, $\Gamma / n_{z}$, for boron determined with 

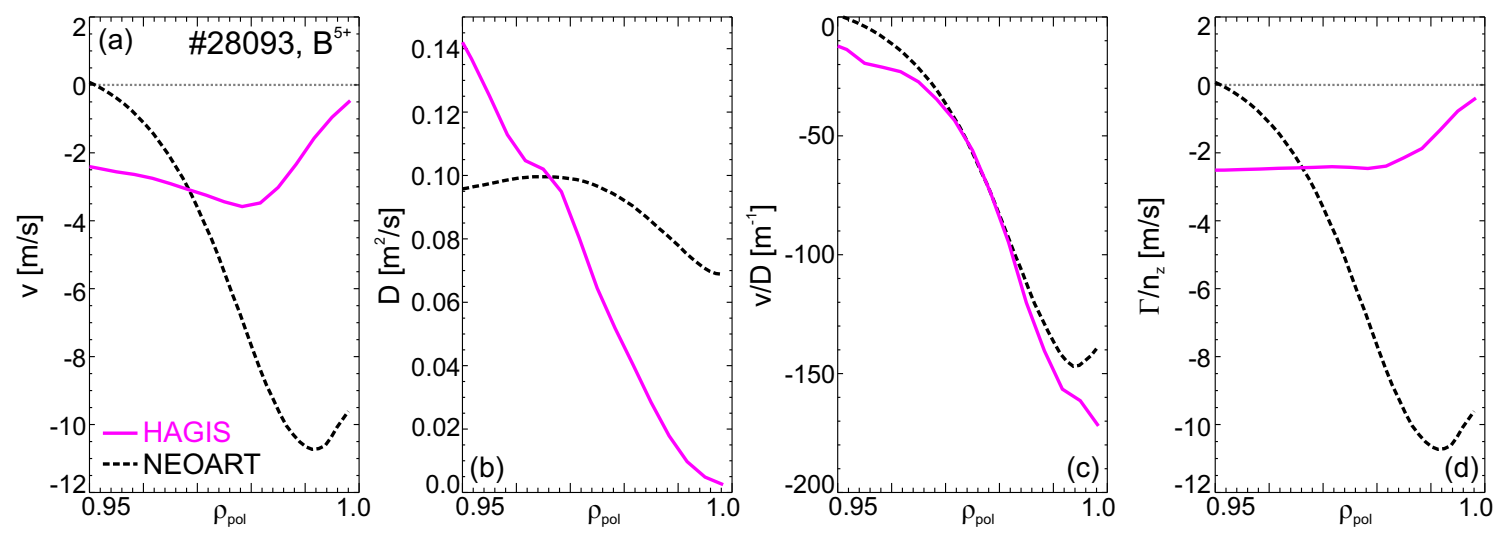

Figure 13: Transport coefficients and impurity flux of of $\mathrm{B}^{5+}$ obtained with NEOART and HAGIS: (a) convective velocity $v$, (b) diffusion coefficient $D$, (c) drift parameter $v / D$ and (d) impurity flux normalized to the density, $\Gamma / n_{z}$.

NEOART, which implies poloidally symmetric density profiles, in black. The magenta curves represent the transport coefficients and the impurity flux evaluated with HAGIS, which includes the impurity asymmetry. Here, the values for $D$ and $v$ are obtained from a pair of HAGIS simulations, one of which has constant density initially in order to get a different density gradient. The fluxes are then equated to $-D \nabla n+n v$ for both calculations. Note that there are no particle sources in the HAGIS simulations. As shown in figure 13 , the $v, D$ and $\Gamma / n_{z}$ profiles are reduced in the edge pedestal, while the drift parameter $v / D$, which determines the gradient of the particle density, is very similar to the poloidally symmetric case calculated with NEOART.

Comparing the experimentally determined $v / D$ to the neoclassical prediction using NEOART shows quantitative agreement within the measurement uncertainties [10]. This demonstrates that the poloidal rearrangement of the impurities does not lead to a significant deviation of the impurity peaking from the conventional neoclassical level, consistent with previous studies [2].

\section{Summary and Conclusions}

An edge rotation database was developed at ASDEX Upgrade to study the flow behaviour in H-mode plasmas with different collisionalities and different main ion species (D, $\mathrm{H}$ and $\mathrm{He}$ ). In the presented discharges the main ion collisionality at the pedestal top varied between 0.18 and 11.5. Comparison of the measured poloidal flows to neoclassical theory shows good agreement in all cases. Below a certain collisionality threshold, neoclassical theory predicts that $v_{\theta, i}$ flips sign from the electron to the ion diamagnetic direction. Simultaneously, the measured edge impurity toroidal rotation is shifted to the counter-current direction. Using radial force balance, $v_{\phi, i}$ is derived from the impurity measurements, the measured electron density profile and from the neoclassically predicted $v_{\theta, i}$. The main ion toroidal flow shows a weak dependence on collisionality and remains co-current, since the differential toroidal rotation between 
main ions and impurities scales with collisionality and reaches values of up to $30 \mathrm{~km} / \mathrm{s}$ at low collisionality $\left(\nu_{*, i} \approx 0.2\right)$. The reason is that at low collisionality friction between main ions and impurities becomes less efficient. The main mechanism determining $v_{\phi, i}$ at the plasma edge remains an open issue and is subject to future work.

Analysis of the impurity flow structure on the flux surfaces shows that the poloidal asymmetry in the edge flow pattern is consistent with the continuity equation when taking into account the poloidally asymmetric impurity density distribution. Direct measurements of the impurity density at the HFS and LFS reveal the existence of an in-out impurity asymmetry, with an impurity localization at the HFS of up to a factor of $\sim 3$. Despite the presence of a poloidal impurity density asymmetry, the ion temperature and the electrostatic potential are found to be simultaneous flux functions within the measurement uncertainties. The measured flows have been compared to theoretical predictions based on the parallel momentum balance. The in-out asymmetry is caused by an interplay of all terms in the parallel force balance with friction between main ions and impurities and the poloidal centrifugal force playing an important role close to the plasma boundary. The key features of the experimental data including the shape of the rotation profiles and the poloidal rearrangement of the impurities are reproduced quantitatively by the model.

Including the impurity asymmetries has an impact on the neoclassical fluxes and on the transport coefficients. The convective velocity $v$ and the diffusion coefficient $D$ evaluated with NEOART, which does not take into account the asymmetries, have been compared to those calculated with HAGIS, which includes the asymmetric impurity distribution. The simulations show that $D$ and $v$ separately decrease when including the asymmetry, however, the drift parameter $v / D$ is at the same level as the $v / D$ profile calculated assuming poloidal symmetry. This demonstrates that despite the presence of a poloidally asymmetric impurity density in the edge pedestal, the peaking of the impurity profile can still be computed with conventional neoclassical models, which assume poloidally symmetric impurity distributions.

\section{Acknowledgments}

The authors would like to thank Dr. V. Rozhansky for fruitful discussions. This work has been carried out within the framework of the EUROfusion Consortium and has received funding from the Euratom research and training programme 2014-2018 under grant agreement number 633053. The views and opinions expressed herein do not necessarily reflect those of the European Commission. The support from the EUROfusion Researcher Fellowship programme under grant number WP14-FRFIPP/Viezzer is gratefully acknowledged. F. M. Laggner is a fellow of the Friedrich Schiedel Foundation for Energy Technology. 


\section{References}

[1] H. Biglari et al. Phys. Fluids B, 2:1, 1990.

[2] T. Pütterich et al. J. Nucl. Mater., 415(1):S334-S339, 2011.

[3] A. V. Chankin et al. Plasma Phys. Control. Fusion, 48:839, 2006.

[4] F. L. Hinton and R. D. Hazeltine. Rev. Mod. Physics, 48(2):239, 1976.

[5] S. P. Hirshman and D. J. Sigmar. Nucl. Fusion, 21(9):1079, 1981.

[6] Y. B. Kim et al. Phys. Fluids B, 3(8), 1991.

[7] K. D. Marr et al. Plasma Phys. Control. Fusion, 52(055010), 2010.

[8] T. Pütterich et al. Nucl. Fusion, 52:083013, 2012.

[9] R. M. Churchill et al. Nucl. Fusion, 53:122002, 2013.

[10] E. Viezzer et al. Plasma Phys. Control. Fusion, 55:124037, 2013.

[11] A. B. Hassam and T. M. Antonsen Jr. Phys. Plasmas, 1:337, 1994.

[12] T. E. Stringer et al. Phys. Rev. Lett., 22:1770, 1969.

[13] A. B. Hassam et al. Phys. Rev. Lett., 66:309, 1991.

[14] J. Kim et al. Phys. Rev. Lett., 72(14):2199, 1994.

[15] F. L. Hinton et al. Phys. Rev. Lett., 72(8):1216, 1994.

[16] R. D. Hazeltine. Phys. Fluids B, 1(10):2031, 1989.

[17] R. E. Bell et al. Phys. Rev. Lett., 81(7):1429, 1998.

[18] W. M. Solomon et al. Phys. Plasmas, 13(056116), 2006.

[19] K. Crombé et al. Phys. Rev. Lett., 95(155003), 2005.

[20] B. A. Grierson et al. Phys. Plasmas, 19:056107, 2012.

[21] B. A. Grierson et al. Nucl. Fusion, 53:063010, 2013.

[22] A. R. Field et al. Plasma Phys. Control. Fusion, 51(105002), 2009.

[23] R. E. Bell et al.. Phys. Plasmas, 17:082507, 2010.

[24] G. Kagan et al. Plasma Phys. Control. Fusion, 53(025008), 2011.

[25] A. Bortolon et al. Nucl. Fusion, 53:023002, 2013.

[26] K. H. Burrell et al. Nucl. Fusion, 54:083020, 2014.

[27] E. Viezzer et al. Nucl. Fusion, 54:012003, 2014.

[28] A. G. Peeters. Phys. Plasmas, 7(1):268, 2000.

[29] S. D. Pinches et al. Comp. Phys. Comm., 111:133, 1998.

[30] A. Bergmann et al. Phys. Plasmas, 8:5192, 2001.

[31] P. A. Schneider et al. Nucl. Fusion, 53:073039, 2013.

[32] E. Viezzer et al. Rev. Sci. Instrum., 83:103501, 2012.

[33] W. Suttrop. Practical Limitations to Plasma Edge Electron Temperature Measurements by Radiometry of Electron Cyclotron Emission. IPP Report 1/306, Max-Planck-Institute for Plasma Physics, Garching, Germany, 1997.

[34] B. Kurzan et al. Rev. Sci. Instrum., 82:103501, 2011.

[35] M. Willensdorfer et al. Rev. Sci. Instrum., 83:023501, 2012.

[36] A. Mlynek et al. Rev. Sci. Instrum., 81:033507, 2010.

[37] E. Viezzer et al. Nucl. Fusion, 53:053005, 2013.

[38] T. Pütterich et al. Phys. Rev. Lett., 102(025001), 2009.

[39] P. Helander and D. J. Sigmar. Collisional Transport in Magnetized Plasmas. $1^{\text {st }}$ edition, ISBN 0-521-80798 -0, Cambridge University Press, Cambridge, 2002.

[40] R. Dux and A. G. Peeters. Nucl. Fusion, 40(10):1721, 2000.

[41] U. Stroth et al. Plasma Phys. Control. Fusion, 53:024006, 2011.

[42] R. M. McDermott et al. Phys. Plasmas, 16:056103, 2009.

[43] C. Angioni and P. Helander. Plasma Phys. Control. Fusion, 56:124001, 2014.

[44] T. Fülöp and P. Helander. Phys. Plasmas, 6:3066, 1999.

[45] L. C. Ingesson et al. Plasma Phys. Control. Fusion, 42:161, 2000.

[46] Ye O. Kazakov et al. Plasma Phys. Control. Fusion, 54:105010, 2012. 
[47] M. L. Reinke et al. Plasma Phys. Control. Fusion, 54:045004, 2012.

[48] T. Pütterich et al. Plasma Phys. Control. Fusion, 55:124036, 2013.

[49] C. Angioni et al. Nucl. Fusion, 54:083028, 2014.

[50] F. J. Casson et al. Plasma Phys. Control. Fusion, 57:014031, 2015.

[51] R. M. Churchill et al. Rev. Sci. Instrum., 84:093505, 2013.

[52] B. LaBombard. KN1D User Manual. http://www.psfc.mit.edu/ labombard/KN1D_Source_Info.html, 2001.

[53] R. M. Churchill et al. Phys. Plasmas, 22:056104, 2015.

[54] C. Theiler et al. Nucl. Fusion, 54:083017, 2014.

[55] T. Fülop and P. Helander. Phys. Plasmas, 8:3305, 2001.

[56] F. L. Hinton and S. K. Wong. Phys. Fluids, 28:3082, 1985.

[57] F. J. Casson et al. Phys. Plasmas, 17:102305, 2010.

[58] J. A. Wesson. Nucl. Fusion, 37:577, 1997.

[59] L. Guazzotto et al. Phys. Plasmas, 12:056107, 2005.

[60] V. Rozhansky et al. Nucl. Fusion, 55:073017, 2015.

[61] V. Rozhansky. Sov. Journ. Plasma Phys., 5:771, 1979. 